(B) ALTERNATIVE APPROACHES 


\title{
MAJOR OBSERVATORIES VERSUS ECONOMY-CLASS OBSERVATORIES IN SPACE
}

\author{
FREEMAN J. DYSON \\ Institute for Advanced Study, Princeton, $N J$
}

\begin{abstract}
Big space-instruments are essential for observing fine details of selected objects. Small space-instruments are essential for scanning large areas of sky, for discovering and cataloguing new objects, and for monitoring variable or transient sources of radiation. If astronomy is to flourish, both types of instrument must be provided. The problem is not to choose between big and small. The problem is to find the optimum mixture of big and small. Various examples from past history and from future plans illustrate the importance of maintaining a variety of different types of instrument.
\end{abstract}

\section{Baade and Zwicky}

The title of this talk was imposed on me by the organizers of the meeting. I did not choose it. I do not like it. The word "versus" implies an antogonism between large and small space-telescopes. No such antagonism ought to exist. Any reasonable program for space-based astronomy must have large and small instruments working in collaboration. Large and small are needed for different jobs. They should complement each other, not comete with each other. Lyman Spitzer wrote in his 1968 article describing the work of the Large Space Telescope: "Quite apart from the engineering desirability of launching smaller space telescopes before building the large instrument, the astronomical requirement for a continuing series of smaller space telescopes should be an overriding consideration in setting the pace of the Large Space Telescope effort." Now it is the job of the IAU to make sure that, in the euphoria surrounding the launch of the Hubble Telescope, these words of Spitzer are not forgotten.

Before looking at future missions I go back to the past. A story from the past may help us to avoid mistakes in the future. I go back fifty years, to the beginning of the Palomar observatory.

The 18-inch Schmidt was the first telescope to be put on Palomar mountain. It was working there for ten years before the 48-inch Schmidt and the 200-inch Hale Telescope arrived. It was there because Fritz Zwicky was interested in supernovae. Zwicky was the first astronomer to observe supernovae systematically. To observe a significant number of supernovae, Zwicky needed a telescope that he could have all to himself, taking pictures of galaxies night after night. He organized the building of the 18-inch Schmidt on Palomar and made sure that he would be in charge. To have unlimited observing time was more important than to have a big mirror. Zwicky made good use of his time. Here is his own account written thirty years later:

"I put this instrument into operation on the night of September 5, 1936, and immediately started a survey of several thousand galaxies. Twenty supernovae were

Y. Kondo (ed.), Observatories in Earth Orbit and Beyond, 399-405.

(C) 1990 Kluwer Academic Publishers. Printed in The Netherlands. 
discovered by my assistant J.J. Johnson and myself in the period from 1936 to 1941. At the same time much of the observational material was gathered for our six-volume catalogue of galaxies and clusters of galaxies. For the construction of the 18-inch Schmidt telescope, its housing, a full-size objective prism, a small remuneration for my assitant, and the operational costs for the whole project during ten years, only about fifty thousand dollars were expended. This probably represents the highest efficiency, as measured in results achieved per dollar invested, of any telescope presently in use, and perhaps of any every built, with the exception of Galilei's little refractor."

Even after making allowances for Zwicky's notorious egotism, I have to agree with his claims for the 18-inch Schmidt. His exploration of the world of the supernovae was of immense importance to the future of astronomy. His sky-survey set the style for many later surveys done with bigger instruments and bigger investments of man-power and money. He was the first astronomer to search deliberately for the most violent events in the universe, and he was the first to understand that if you want to search efficiently for violent events you must cover the whole sky repeatedly. Insofar as the modern style in astronomy is to concentrate attention on short-lived and violent processes, Zwicky was the first modern astronomer. And the 18-inch Schmidt gave him the chance to show what he could do. Among other things, he discovered in his survey of clusters of galaxies the first clear evidence that the universe contains missing mass.

Here is another quote from Zwicky's autobiography:

"Astronomers expect that photoelectronic devices of small aperture and light weight will eventually outperform the giant 200-inch Hale reflector on Palomar mountain. In any case these new instruments will be indispensable if we intend seriously to explore the universe with the aid of rocket-borne instrumentation."

Zwicky was wrong to claim that small telescopes with electronic detectors would put the 200-inch out of business. Zwicky was blinded by his dislike of Walter Baade, the chief observer at the 200 -inch in those days. When Zwicky wrote that small electronic telescopes would outperform the 200 -inch, what he had in mind was that Zwicky would outperform Baade. Both of them were great astronomers. Neither of them outperformed the other. Zwicky was a cantankerous genius, quick in thinking of usefule schemes and energetic in carrying them out. Baade was an artist, a virtuoso performer who played the 100-inch and 200-inch telescopes like violins. His great contribution to astronomy was the identification of the two populations of stars. The decisive step in his discovery of Population II was to resolve into stars the great red blob at the center of M31. Baade was able to see these stars only by pushing the 100-inch telescope to the limit of its capabilities. For this purpose, any smaller telescope would have been useless.

Once in their lives, when Zwicky and Baade were both young and before they had become enemies, they wrote together a theoretical paper of extraordinary originality. Their paper appeared in 1934, just two years after Chadwick had discovered the neutron. At the end of their paper, Baade and Zwicky said: "With all reserve we advance the view that a supernova represents the transition of an ordinary star into a neutron star, consisting mainly of neutrons. Such a star may possess a very small radius and an extremely high density." 
These remarks of Baade and Zwicky were ignored by astronomers for 33 years, until neutron stars were discovered by radio-astronomers. Now we know that almost everything Baade and Zwicky said in their 1934 paper was true. It was a great loss of science that they did not continue their collaboration. Each of them alone was great, but together they would have been greater. If they had remained friends, neutron stars might have been discovered twenty-five years sooner, in 1942 instead of 1967 . It happened that in 1942 Baade used the 100-inch to take the classic pictures of the Crab Nebula which you have all seen. He identified the peculiar star near the center of the nebula, which he suspected of being the stellar remnant of the supernova that exploded in 1054 A.D. According to the Baade-Zwicky paper of 1934, the stellar remnant ought to be a neutron star. Baade asked his friend Rudolf Minkowski to take a spectrum. Minkowski, also using the 100-inch, took a spectrum of the star and found it completely featureless with no lines at all. Minkowski calculated the temperature of the star and found it to be 500000 degrees. The spectrum made it certain that this was the supernova remnant, a weird and unique object. But Baade and Minkowski did not go further. They did not look at the star again. They did not in their 1942 publications mention the possibility that it might be a neutron star. Perhaps by 1942, Baade had come to consider a neutron star to be merely one of Zwicky's crazy ideas from which he was glad to dissociate himself. From a human point of view, such a reaction is understandable. But from a scientific point of view, it was a great opportunity missed.

As Cocke, Disney and Taylor discovered, with a one-meter telescope at Steward Observatory in 1969, the star in the Crab Nebula flashes on and off thirty times a second. A few years later, a graduate student observed the flashes and measured their changing period with a one-meter telescope on the Princeton Campus. Whatever a Princeton student did with a one-meter telescope under the polluted sky of New Jersey, Zwicky could have done with his 18-inch under the dark sky of Palomar thirty years earlier.

Zwicky was uniquely qualified to be the discoverer of neutron stars. He was one of the few astronomers in the 1940's who took neutron stars seriously. He was one of the few who saw the potential of electronic photo-detection. And he was one of the few who had unlimited observing time on a good small telescope. If he had put his mind to the problem, he might have seen the star in the Crab Nebula flashing in 1942, and the whole subsequent history of astronomy would have been changed. He missed his chance because he was not talking to Baade.

I told this story of Baade and Zwicky at some length, because it illustrates my general theme. The moral of the story is that you miss making important discoveries if you work with big telescopes alone or with small telescopes alone. You need a big telescope to get good spectra of faint objects. You need a small telescope to search the sky for objects you did not know existed. You need a big telescope to detect objects out to the extreme limits of faintness. You need a small telescope to keep watching a variable object, to see how it changes from night to night or from millisecond to millisecond. But it is not enough to have both big and small telescopes. You need also to have big-telescope astronomers and small-telescope astronomers talking to each other. Zwicky was a classic case of a small-telescope astronomer doing great work with limited means. Baade was a classic big-telescope 
astronomer, searching the far distances of the universe until, as Hubble said, "At the last dim horizon we search among ghostly errors of observation for landmarks that are hardly more substantial". I won't attempt to apportion the blame between Zwicky and Baade for the fact that they stopped being friends. Both were to blame for missing their chance to put their combined talents to work to solve the mystery that lies at the heart of the Crab Nebula.

\section{HST and IUE}

I now jump 48 years from 1942 to 1990 . We shall have, if all goes well, two ultraviolet telescopes observing from orbit in 1990, HST and IUE. In many respects, the history of the 1940's will be repeating itself. HST is not quite as big as the 200inch, but it is an enterprise of the same character, massive in scale and cost, plagued by technical difficulties and delays, supported by an effective public-relations campaign, and giving us finally a huge extension of our view of the far reaches of the universe. IUE is not quite as cheap as Zwicky's 18-inch Schmidt, but it is exactly as small, a little half-meter mirror sitting in the sky, unnoticed by the public, pouring our important and unimportant astronomical observations for twelve years while its big sister was still struggling to be born. In one respect history has changed. For better of for worse, the management of telescopes has passed from individuals to committees. No cantankerous genius like Fritz Zwicky is in charge of IUE. IUE is run by an international consortium with input from large numbers of users in many countries. And HST is run by a big organization in Baltimore with input from almost everybody. With luck, IUE may stay alive for another five years. But it is living on borrowed time, its 14-year-old Vidicon detectors have long been obsolete, and it would be ready for retirement if we had a more modern IUE ready to replace it.

The Hubble telescope will not be a replacement for IUE. The two instruments complement each other without much overlapping. HST is needed for deep exploration and high-resolution imaging. IUE is needed for rapid spectroscopy and wide coverage of brighter objects. HST will discover a vast number of things that IUE could not reach. But the greater outreach of HST comes at a high cost in observing time. If you observe with HST at the limit of its capabilities, you need to wait a long time to collect the photons. Since most of the interesting in the sky are faint, HST will never have enough time to observe as many of them as we would wish. The observing time of HST will always be over-subscribed, and no time will be allotted for looking at things which could just as well be observed with IUE. That is the reason why HST cannot be a replacement for IUE.

I jump back again to the past to see what we ought to be doing to replace IUE. Zwicky's 18-inch Schmidt was, like IUE, in need of replacement after 10 years of productive work. Both IUE and the little Schmidt were unnecessarily small for the work they had to do. The replacement for the little Schmidt was not the 200-inch, just as the replacement for IUE is not HST. The replacement for the little Schmidt was the 48-inch Schmidt, which was installed then years after the little Schmidt at Palomar and put to work on the Palomar Sky Survey with Hubble himself in charge. What we need to replace IUE now is the space-based equivalent of the 48- 
inch Schmidt. The replacement for IUE does not need a 48-inch mirror. I imagine it as a small ultraviolet telescope with tenth-of-a-second-of-arc resolution and modern CCD detectors, weighing and costing about one-fifth as much as HST. Ted Stecher proposed such a telescope as an Explorer mission a few years ago. NASA turned it down. His telescope will soon fly, under the name UIT, as part of the ASTRO shuttle mission. In a ten-day mission it can do some good observing but it cannot replace IUE. If our space-based astronomy program had been driven by scientific rather than political priorities, if the administrators of the program had followed the policy so clearly enunciated by Lyman Spitzer in his 1968 statement, we should have had the replacement for IUE already in orbit several years ago. We should have had a more capable but still modest-sized IUE, exploring the ultra-violet sky and preparing the way for HST, just as the 48-inch Schmidt explored the sky and prepared the way for the 200 -inch fourty years earlier. We could have reconnoitred in depth all the objects which are now candidates for HST observation. We could have picked out and sharpened the crucial questions which only HST can answer. We could have greatly increased the scientific cost-effectiveness of HST by concentrating its precious observing-time upon mysteries that a small telescope could not resolve.

\section{Future Projects}

After giving ten minutes to the past and five to the present, I have ten minutes left for the future. I will not try to mention all possible future missions and arrange them in order of merit. I take it as given that the program of Great Observatory missions recommended by the Greenstein and Field committees, beginning with HST and continuing with GRO for gamma-ray astronomy, SIRTF for infra-red, AXAF for $\mathrm{X}$-rays and LDR for far infra-red and submillimeter astronomy, will sooner or later be launched and flown. These are all splendid missions and I wish them well. I am especially happy to hear that the plan in now to put SIRTF into a high orbit where it will escape from the worst of the miseries that a low orbit imposes on HST. The trouble with the Great Observatory missions is that they kept us waiting too long before they flew. They have had a bad effect on the progress of astronomy, because they discouraged people from building smaller instruments that cost less and could fly sooner. I shall talk briefly about some modest missions that I would like to see flying while we wait for the big ones. One such mission, COBE, went up recently and is doing spectacularly good science. Fortunately, missions comparable in size and quality with COBE need not wait for NASA funding but can now be undertaken by other countries that are active in space-science. The European X-ray and Infra-red satellites ROSAT and ISO will soon be in orbit, filling two of the serious gaps in the NASA program. We are entering an era in which space-based astronomy will be spread among the nations as widely as ground-based astronomy.

I would like to see a series of modest space-telescopes operating in the same manner as IUE, covering all the various parts of the electromagnetic apectrum and acting as survey instruments to complement the various Great Observatories. We should have had at least six small observatories, one for submillimeter, one for infra-red, one for visible, on for ultra-violet, one for extreme ultraviolet and one for X-rays, already up and running. With luck we could build and fly six small 
observatories for the price of one great observatory. Together, they would yield an enormous harvest of science.

Another important class of small space-observatories is concerned with development of new optical technology. The great observatories of the next century will undoubtedly include optical interferometers and large thin mirrors with shapes controlled by active optics. Will we try out these new technologies for the first time in huge billion-dollar instruments? I hope not. We ought already to be planning to launch small interferometric arrays and small thin mirrors with active optical control. These missions would not only give us engineering experience essential for learning how to build bigger ones. They would also be important scientific instruments in their own right, allowing us to observe brighter objects with angular resolution surpassing HST. Once the technology of optical arrays is successfully demonstrated on a small scale, big arrays will probably come to dominate optical space-astronomy just as they have dominated radio-astronomy on the ground. But the radio-astronomers did not jump from single dishes to the VLA in one step. The VLA works well because it grew out of long experience in building and using smaller arrays. If we are wise, we will approach the grand optical arrays of the twenty-first century in the same step-by-step fashion.

As a rule, the cost-effectiveness of small telescopes is greatest if they are designed and used as special-purpose rather than general-purpose instruments. IRAS and COBE were designed for the special purpose of sky survey, and they do only that one job. They have been spectacularly successful because they do one job spectacularly well. Zwicky's 18-inch Schmidt, and its successor the 48-inch Schmidt, were likewise designed for sky survey and were likewise cost-effective.

IUE is an apparent exception to the rule, successful in spite of being a generalpurpose instrument. But even IUE conforms to the rule in so far as it confines its activities to spectroscopy and does not waste time on imaging. Hipparcos is another example of a special-purpose instrument that promises great scientific return from a modest payload. After Hipparcos completes its mission, as I hope it will, there will be other astrometric satellites, also special-purpose, also modest in size and weight, extending the limits of astrometry both in accuracy and in breadth of coverage.

The majority of the small space-telescopes that I have mentioned would be special-purpose instruments designed for sky survey in a variety of wave-bands. Another instrument of this type is a one-meter diffraction-limited telescope dedicated to a digital sky-survey with tenth-of-a-second-of-arc resolution in visible light. The digital sky-survey would be as important to the future of astronomy as the Palomar sky-survey of 1949-1956. It would not be a small mission. It would be intermediate in size and cost between an Explorer mission like COBE and a Great Observatory like HST. It would require considerably larger CCD detectors than are now available. In round numbers, it requires a CCD array with $10^{9}$ pixels to give a one-degree field of view at 0.1 second resolution and to cover the whole sky with thousand-second exposures within a year or two. The data-stream would come to earth at a rate of one megabyte per second and would require a memory of 100 terabytes for permanent storage. These numbers refer to a survey done in a single color. It would be reasonable to plan the mission to do a survey in several colors simultaneously. Then we could have a 3-color survey completed in five years with 
the same bit-rate and 300 terabytes of storage. The numbers are formidable, but my computer-expert friends do not find them unreasonable. A major component of the digial sky-survey enterprise will be learning how to digest and distribute data in usable form from the 300 terabyte storage to astronomers all over the world.

But let us not be carried away by grandiose dreams. The main advantage of small special-purpose space-missions is lost if we let them become too ambitious. We have enough Great Observatoties on the drawing-boards already. What we need now is more small missions, using existing hardware, to fill the gaps between the big ones. Let me end as I began with some good advice from Fritz Zwicky. I quote again from his book, "Discovery, Invention, Research".

"I wish to caution all hotheads that it is not advisable to try to do everything at the same time, a mistake which is often committed by individuals and by institutions whose funds are limited. For instance, the construction of multipurpose telescopes is in general not to be recommended. It is better to concentrate one's attention on specific problems and to build instruments best adapted for their solution."

\section{References}

Spitzer, Lyman Jr.: 1968, Science 161, 225-229

Zwicky, F.: 1969, Discovery, Invention, Research, New York, Macmillan, 91-92, 226

Zwicky, F.: 1948, in Halley Lecture, "Morphological Astronomy", ed(s)., Observatory, 68, 121-143

Baade, W. and Zwicky, F.: 1934, Proc. Nat. Acad. Sci. (USA) 20, 259-263

Baade, W.: 1942, Astrophys. J. 96, 188-198

Minkowski, R.: 1942, Astrophys. J. 96, 199-213

Kondo, Y. (Ed.): 1987, Exploring the Universe with the IUE Satellite, Dordrecht, Reidel, 347 